\title{
Letter
}

\section{Lost in Translation? Measuring Diabetic Neuropathy in Humans and Animals (Diabetes Metab J 2021;45:27-42)}

\author{
Otto Jesus Hernandez Fustes \\ Neurology Service, Complexo Hospital de Clinicas-Universidade Federal do Paraná, Curitiba, Brazil
}

I read with great interest the manuscript by Jin et al. [1], about "Lost in translation? Measuring diabetic neuropathy in humans and animals." This review is an opportunity to discuss diagnostic aspects of a diabetic peripheral neuropathy (DPN) for resident physicians and specialists. The authors describe the functional and structural parameters used as indexes of peripheral neuropathy in preclinical and clinical studies. Nerve conduction studies in large myelinated fibers have been the mainstay of preclinical efficacy screening programs and clinical trials, complemented by sensory tests. Highlighting that a more refined approach is emerging, which incorporates measurements of small fiber density in the skin and cornea along with these traditional trials. We have the following comments and concerns.

I would like to draw your attention to two techniques for assessing small fiber neuropathy (SFN) - the pain-related evoked potentials (PREP) and high-resolution ultrasound (HRU).

The management of people with SFN is a challenge not only for the neurologist or pain specialist but also for the neurophysiologist. The fact of having to find evidence to support clinical complaints when routine studies of nerve conduction are normal has led to the development in recent years of several procedures, neurophysiological, histological and imaging, allowing the advance in diagnosis without yet being considered specific markers.

Neurophysiological methods available to assess possible SFN consist of quantitative sensory test, reflex of the sudomotor axon, sympathetic skin response, autonomic tests and potentials evoked by laser. These techniques are partially invasive, time consuming, expensive and therefore just partially useful in the clinical routine.

PREP might be an alternative because they are a non-invasive, reliable electrophysiological procedure which can assess the signal transmission of A- $\delta$ fibers without large expenditure. PREP detect small fiber dysfunctions in systemic disorders which are associated with generalized polyneuropathies $[2,3]$.

The development and use of new imaging techniques, such as HRU, and electrophysiological techniques, like PREP, without a doubt add a new stair step to the diagnosis and management of neuropathies and in particular SFN, in addition to facilitating more comprehensive studies that allow us to achieve more reliable conclusions [3].

I agree with the authors when they state that the translation of the preclinical promise for the clinical approval of therapies targeting diabetic neuropathy in the past 50 years has been fraught with flaws, with the blame equally distributed between the design of imperfect drugs, the preclinical models and clinical trial designs. We need a multidisciplinary approach to a multidisciplinary problem, such as DPN, from basic studies to a better determination of pathogenesis.

We congratulate the authors for this, and the opportunity to delve into a topic so interesting.

\section{CONFLICTS OF INTEREST}

No potential conflict of interest relevant to this article was reported.
Corresponding author: Otto Jesus Hernandez Fustes

(iD https://orcid.org/0000-0003-0778-5376

Neurology Service, Complexo Hospital de Clinicas-Universidade Federal do Paraná, Av.

Marechal Floriano 170, sala 1509, Curitiba 80090-020, Brazil

E-mail: otto.fustes@hc.ufpr.br
This is an Open Access article distributed under the terms of the Creative Commons Attribution Non-Commercial License (https://creativecommons.org/licenses/by-nc/4.0/) which permits unrestricted non-commercial use, distribution, and reproduction in any medium, provided the original work is properly cited. 


\section{REFERENCES}

1. Jin HY, Moon SS, Calcutt NA. Lost in translation? Measuring diabetic neuropathy in humans and animals. Diabetes Metab J 2021;45:27-42.

2. Fischer M, Hoffken O, Ozgul OS, Maier C. Bilaterally pro- longed latencies of pain-related evoked potentials in peripheral nerve injuries. Neurosci Lett 2018;684:78-85.

3. Hernandez Fustes OJ. Focus on nerve fiber type: a diagnostic strategy for diabetic polyneuropathy. J Diabetes Investig 2021; 12:459-60. 